\title{
The DAMPE experiment: first data from space
}

\author{
Ivan De Mitri ${ }^{1,2, a}$ (on behalf of the DAMPE Collaboration) \\ ${ }^{1}$ Dip.to di Matematica e Fisica "E. De Giorgi", Università del Salento, Via per Arnesano, I-73100, Lecce, Italy \\ ${ }^{2}$ Istituto Nazionale di Fisica Nucleare (INFN), Sezione di Lecce, Via per Arnesano, I-73100, Lecce, Italy
}

\begin{abstract}
The DAMPE satellite has been successfully launched in orbit on December 2015. The science goals of the mission include the study of high energy cosmic ray electrons, photons, protons and nuclei in a wide energy range: $10^{9}-10^{14} \mathrm{eV}$. A report on the mission status will be presented, together with on-orbit detector performance and first data coming from space.
\end{abstract}

\section{Introduction}

The DAMPE (DArk Matter Particle Explorer) satellite was launched on December 17, 2015 and is in smooth data taking since few days after. Data from various subdetectors allow an efficient identification of the electron signal over the large (mainly proton-induced) background. As a result, the all-electron spectrum will be measured with excellent resolution from few $\mathrm{GeV}$ up to few $\mathrm{TeV}$, thus giving the opportunity to cleary observe a cutoff and/or identify a possible contribution of nearby sources. Moreover the study of cosmic ray (CR) electrons and high energy photons will allow an indirect search for dark matter signatures. Even if primarily optimized to collect electrons and gammas, DAMPE provides good tracking, calorimetric and charge measurements also in case of protons and nuclei. This will allow precise measurement of CR protons and nuclei energy spectra from few $\mathrm{GeV}$ up to about hundreds $\mathrm{TeV}$. The high energy region will be explored with better precision compared to previous experiments: spectral indexes for individual species could then be well measured and the observed hardenings around $250 \mathrm{GeV} / \mathrm{n}$ could be studied and better quantified.

\section{The DAMPE detector}

DAMPE consists of a Plastic Scintillator strip Detector (PSD) that serves as anti-coincidence and charge detector, a Silicon-Tungsten tracKer (STK) to reconstruct the direction (and charge) of incident particles, a BGO imaging calorimeter (BGO) of about 32 radiation lengths that measures the energy with high resolution and distinguishes between electrons and protons, and a NeUtron Detector (NUD) that can further increase the hadronic shower rejection power (see Fig. 1).

The PSD is designed to measure the charge $(Z)$ of incident high-energy particles, up to $Z=26$, by looking at the energy release due to ionization process, that is approximately proportional to $Z^{2}$. It has a $825 \times 825 \mathrm{~mm}^{2}$ active area made by organic plastic scintillators. Only light materials have been

\footnotetext{
a e-mail: ivan.demitri@le.infn.it
} 

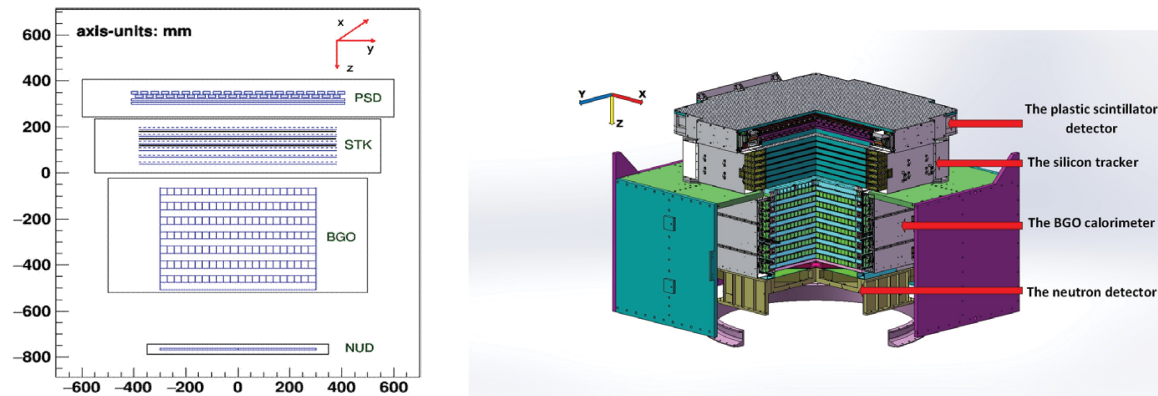

Figure 1. Structure diagram (left) and 3D view (right) of the DAMPE detector.

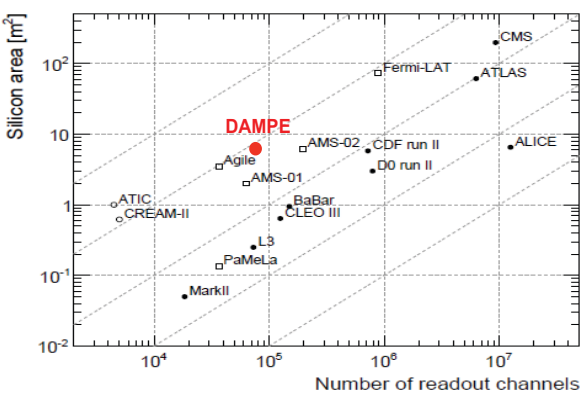

Figure 2. Comparison of the DAMPE STK features with other detectors.

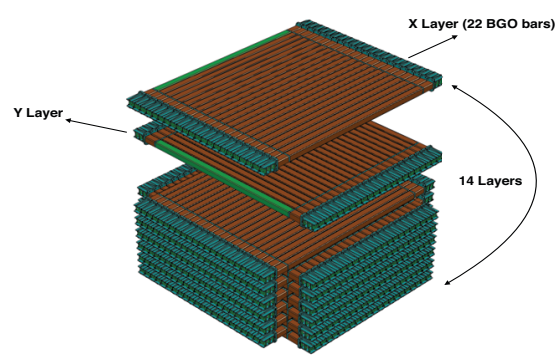

Figure 3. 3D view of the DAMPE BGO scintillator calorimeter.

used in the mechanical structure, in order to avoid initiating electromagnetic showers before the STK. It is arranged in a double layer configuration and has in total 82 detector modules. Each module is made by a long plastic scintillator bar of $825 \mathrm{~mm}$ with a $28 \times 10 \mathrm{~mm}^{2}$ cross section and is read out by two PMTs at each end. The modules are staggered by $8 \mathrm{~mm}$ in the layer, and the bars in the two layers are perpendicular, resulting in a overall efficiency of $99.75 \%$. A double dynode readout for each PMT has been implemented in order to cover a wide range, namely from 0.1 to 1400 times the energy released by a Minimum Ionizing Particle (MIP), with an overlap region for calibration.

The Silicon-Tungsten tracKer is devoted to the precise reconstruction of the particle track. It is made by twelve position-sensitive silicon detector planes (six planes for the $x$-coordinate, six planes for the $y$-coordinate). Three layers of tungsten are inserted in between the silicon $x-y$ planes (2, 3 , and 4) to convert gamma rays in electron-positron pairs. The specifications of STK are given in Table 1 and a comparison with other experiments is shown in Fig. 2 looking at active area and number of channels. The tracking resolution is expected to be $0.2^{\circ}$. The STK analog readout can also provide an independent measurement of the incident particle charge, at least up to medium mass nuclei. It is a compact system where readout and power supply electronics are mounted on the sides [2, 5].

The BGO calorimeter is devoted to measure the energy deposition of incident particles and to reconstruct the shower profile. Moreover the trigger of the whole DAMPE system is based on the signals from the BGO and the shower profile is fundamental to distinguish between electromagnetic and hadronic showers. The calorimeter is composed of $308 \mathrm{BGO}$ crystal bars $\left(2.5 \times 2.5 \times 60 \mathrm{~cm}^{3}\right.$ is the volume of each single bar). The crystals are optically isolated from each other and are arranged 
Table 1. Main STK specifications.

\begin{tabular}{ll}
\hline Active area of each silicon layer & $0.5534 \mathrm{~m}^{2}$ \\
Silicon thickness & $320 \mu \mathrm{m}$ \\
Silicon strip pitch & $121 \mu \mathrm{m}$ \\
Tungsten thickness & $1 \mathrm{~mm}$ \\
Depth (rad. lengths) & 0.976 \\
Power consumption & $82.7 \mathrm{~W}$ \\
Mass & $154.8 \mathrm{~kg}$ \\
\hline
\end{tabular}

Table 2. Main BGO specifications.

\begin{tabular}{ll}
\hline Active area & $60 \times 60 \mathrm{~cm}^{2}$ \\
Depth (rad. len.) & 32 \\
Sampling & $>90 \%$ \\
Long. segmentation & 14 layers \\
Lat. segmentation & $\sim 1$ Molière radius \\
\hline
\end{tabular}

horizontally in 14 layers each made by 22 bars (Fig. 3). The bars of a layer are ortogonal to those of the following one in order to reconstruct the particle path in both views $(x-z$ and $y-z)$. The total vertical depth of the calorimeter is about 32 radiation lengths and 1.6 nuclear interaction lengths. This unprecedented depth ensures that almost $100 \%$ of the energy of electrons and $\gamma$-rays is deposited in the calorimeter, and about $40 \%$ in the case of protons anad nuclei. The high energy limits of the DAMPE measurement ranges (about few $\mathrm{TeV}$ for electrons/photons and hundreds $\mathrm{TeV}$ for nuclei, depending on the their fluxes) are manly determined by its very large geometric factor, which is about $0.3 \mathrm{~m}^{2} \mathrm{sr}$ for hadrons and even larger for electrons/photons. Table 2 summarizes the key parameters of BGO calorimeter. Each crystal element is read out by two PMTs, mounted on both ends of the crystal. The measured light asymmetry allows to further estimate where the energy has been deposited along the BGO bar. In order to cover a very large dynamic range $\left(2 \times 10^{6}\right)$, the signals are collected from three different dynodes covering different ranges on each side.

Typically hadron-induced showers produce roughly one order of magnitude more neutrons than electron-induced showers. The neutrons quickly thermalize in the BGO calorimeter and their activity can be detected by a suitable NeUtron Detector (NUD, see Fig. 1) within few $\mu$ s, thus providing a further tool to distinguish the types of high-energy showers. The NUD is made by four boron-loaded plastics, each with a set of PMTs and related electronics. Indeed neutrons entering the boron-loaded scintillator undergo a capture process (namely ${ }^{10} B+n \rightarrow{ }^{7} L i+\alpha+\gamma$ ), whose probability is inversely proportional to their velocity, within an average capture time that is inversely proportional to the ${ }^{10} B$ loading. The scintilators are wrapped with a layer of aluminium film for photon reflection, and anchored in an aluminium alloy framework. The space between plastic scintillators and the framework ( $1 \mathrm{~mm}$ on each side) has been filled with silicone rubber to relieve the vibration during the launch of the satellite.

\section{Ground tests and on-orbit operation}

An extensive Monte Carlo simulation activity was done during R\&D phase in order to find a proper compromise between physics goals and limitations on geometry, power consumption and weight. DAMPE performances were verified by a series of beam tests at CERN with electrons, photons, pions, protons and nuclear fragments. The wide energy range of the electron beams and the high-purity of the proton beams were sufficient to check energy resolution (Fig. 4), linearity and $e / p$ separation. Also a beam of argon fragments was used for testing the detector response to nuclei. Details of the test-beam results as well as the features of the qualification module can be found in [1-6]. Furthermore many tests (namely energy response to MIPs, efficiencies, detector alignment, etc.) have been performed during different stages of the DAMPE assembly using a large samples of CR mouns at ground.

After launch, the spacecraft entered a sky-survey mode immediately and a dedicated-calibration of the detector was performed in two weeks. The calibration included the determination of pedestals, 

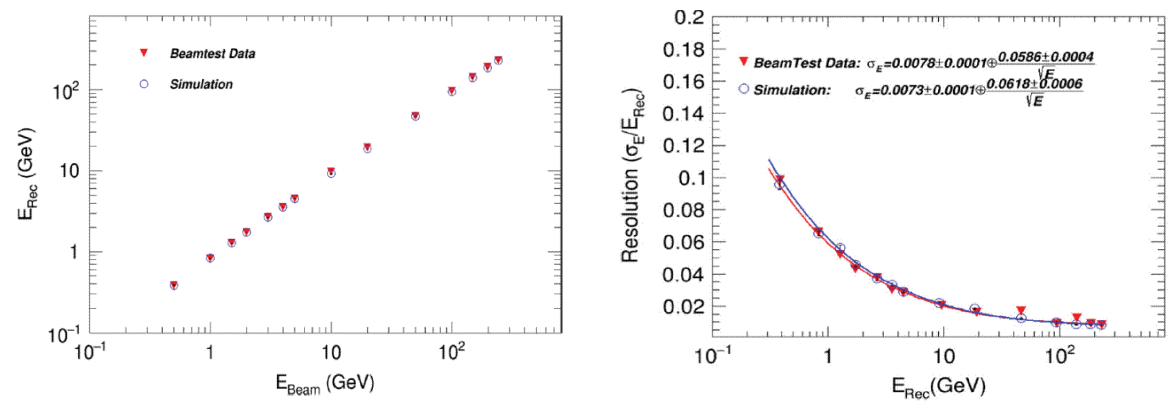

Figure 4. Linearity and resolution of the DAMPE BGO calorimeter for electromagnetic showers: comparison of beam test data with simulations [3].

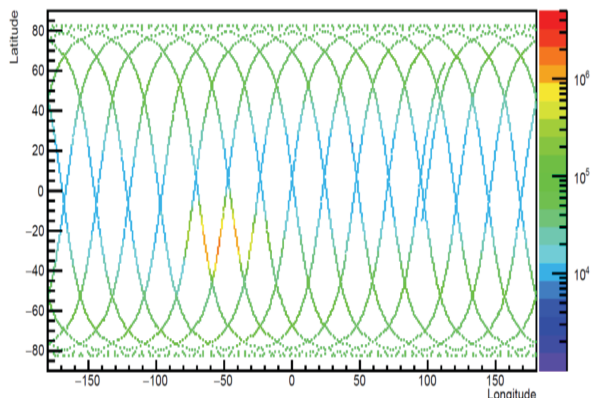

Figure 5. Detected counting rate vs latitude and longitude (arbitary units on the color palette). The South Atlantic Anomaly is clearly visible.

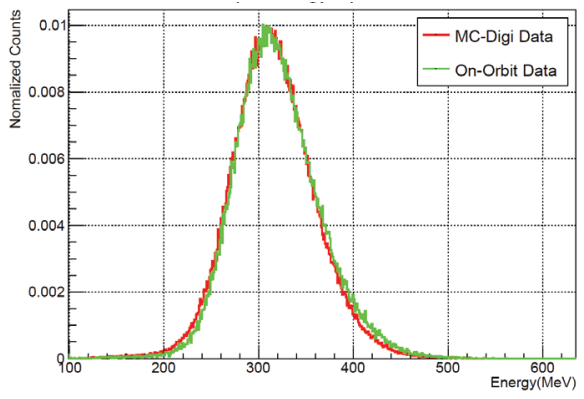

Figure 6. MIP peak in the BGO calorimeter: comparison of simulation with on-orbit data.

response to MIPs, alignment, and timing. By comparing on-orbit data with simulations and on-ground tests we can conclude that the detector is working very well. The satellite is on a solar-synchrous orbit lasting 95 minutes. The pedestal calibration is performed twice per orbit and the global trigger rate is kept at $\sim 60 \mathrm{~Hz}$ by using different pre-scale for unbiased and low-energy triggers at different latitudes (see Fig. 5 and [2]). Data are packaged with timestamp and transmitted to ground at a rate of about 5 millions events per day corresponding to $\sim 15 \mathrm{~GB} /$ day, that increases up to $\sim 100 \mathrm{~GB} /$ day after offline reconstruction.

\section{First on-orbit data and expected performances}

The DAMPE detector started taking physics data very soon after the launch. The performance parameters (temperature, noise, spatial resolution, efficiency) are very stable and very close to what expected. The absolute energy measurement has been checked by using the geomagnetic cut-off and it results well calibrated. Also the absolute pointing has been successfully verified. Indeed the photon data collected in 165 days were enough to draw a preliminary high-energy sky-map where the main gamma-sources are visible in the proper positions. Fig. 6 shows the MIP peak reconstructed with the energy released in the BGO crystals. It is evident the agreement with the distribution derived from the simulation. The energy released in the PSD allows measuring the particle charge and distinguishing the different nuclei in the CR flux. Figs. 7 and 8 show preliminary results of these measurements for 


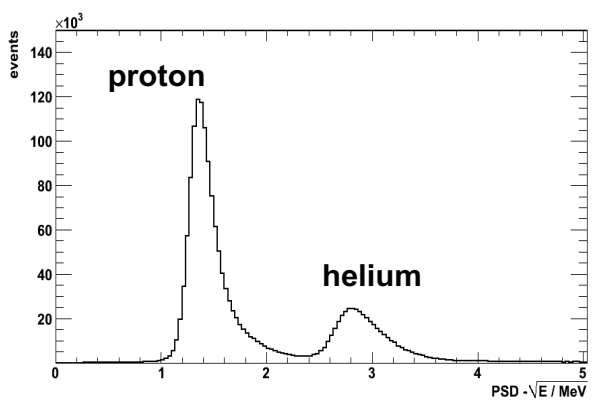

Figure 7. Preliminary distribution for light CR component as measured in the DAMPE PSD.

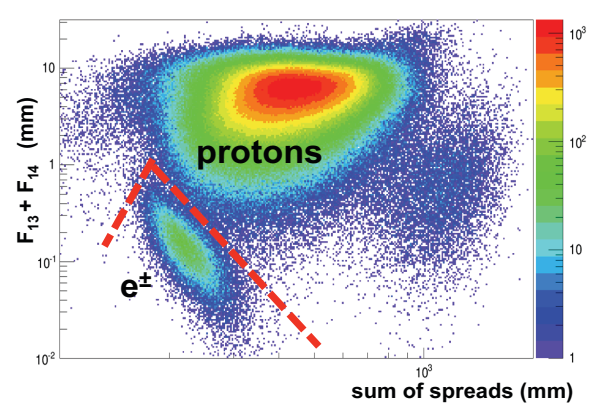

Figure 9. Preliminary result about e/p separation (see text). The background on the right is due to CRs entering the satellite from the sides.

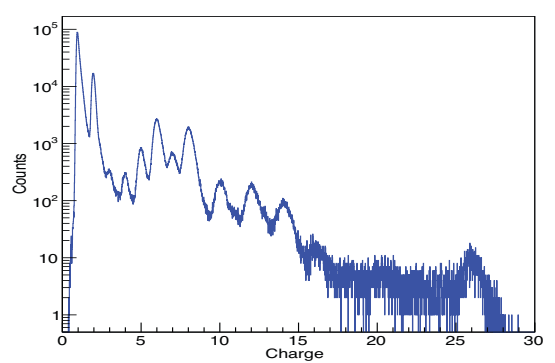

Figure 8. Preliminary $Z$ measurement up to iron with the DAMP PSD.

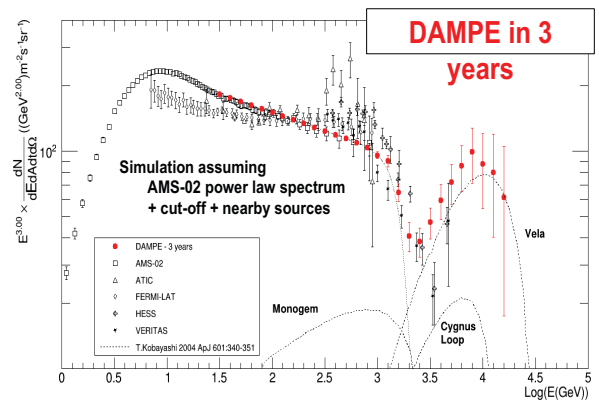

Figure 10. All-electron spectrum. The red dots represent the possible DAMPE measurement in 3 years.

the light component ( $H$ and $H e$ ) and for the full range up to iron $(1 \leq Z \leq 26)$, respectively, for a first sample of on-orbit data.

The measurement of the electron (plus positron) flux is one of the main goal of the DAMPE mission. The shower development in the BGO provides the tool to distinguish leptons from hadrons. Among others, a shape parameter has been defined: $F_{i}=\operatorname{spread}_{i} \times \frac{E_{i}}{E_{t o t}}$, where $i$ is the index of the BGO layer $(1 \leq i \leq 14)$, spread $_{i}$ is the shower width in a given layer, $E_{i}$ and $E_{t o t}$ are the energy on the single $i$-th layer and on all the calorimenet, respectively. Using the shape parameters on the last BGO layers $(13,14)$ it is possible to separate leptons from hadrons with a very large rejection power (preliminary results in Fig. 9). The rejection capability will be further enhanced by means of the NUD.

The DAMPE detector is expected to take data for more than 3 years. This time interval is sufficient to deeply investigate many open questions in galactic CR studies. In Fig. 10 the possible DAMPE measurement of the so called "all-electron" spectrum in 3 years is shown. Given the excellent resolution, spectral features like a possible cut-off at about $1 \mathrm{TeV}$ or a flux increase due to nearby astrophysical sources or dark matter signatures [7], could be well detected.

Many experiments [8-11] observed a discrepant hardening of the CR elemental spectra at about $250 \mathrm{GeV} / \mathrm{n}$. This is another interesting topic related to CR origin and propagation. Due to its very large geometric factor, DAMPE will be able to significantly reduce statistical uncertainties in the break region and give a precise measurements on the high energy spectral index of each nuclear 

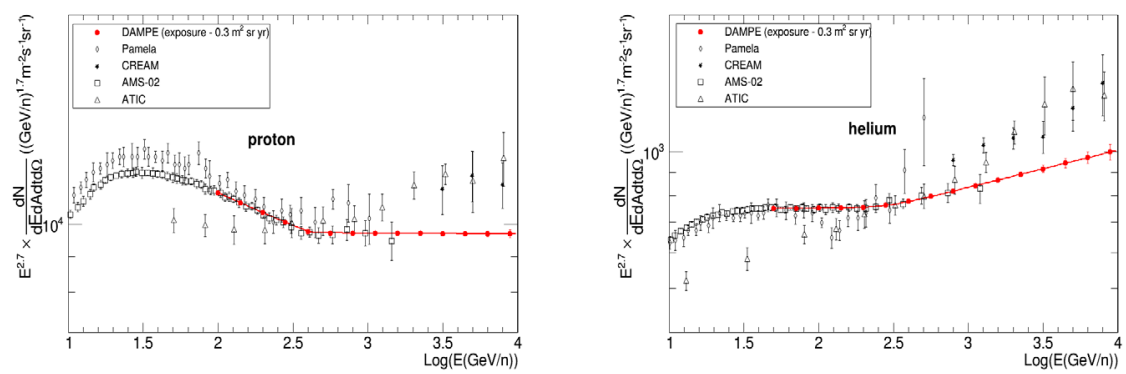

Figure 11. Proton (left) and helium (right) spectra in the range $10 \mathrm{GeV}-10 \mathrm{TeV}$. The red dots indicate the possible DAMPE measurement (for an exposure of $0.3 \mathrm{~m}^{2} \mathrm{sr} \mathrm{yr}$ ) assuming the AMS-02 data fit and its extrapolation to high energy.

species (see Fig. 11). Moreover the large exposure will allow extending energy spectra measurements for protons and nuclei up to hundreds TeV. This also apply quantities like the boron-to-carbon ratio, that could be well measured up to about $10 \mathrm{TeV} / \mathrm{n}$.

\section{Conclusions}

The DArk Matter Particle Explorer (DAMPE) is a space mission supported by the strategic space projects of the Chinese Academy of Sciences with the contribution of Swiss and Italian (namely INFN and MIUR) institutions. The rocket has been successfully launched on December 17, 2015 and DAMPE presently flies regularly on a sun-synchronous orbit at the altitude of about $500 \mathrm{~km}$. Four different detectors are arranged on the satellite: a plastic scintillator array (PSD), a silicon-tungsten tracker (STK), a BGO calorimeter and a neutron detector (NUD). They are devoted to measure the fluxes of charged CRs (electrons, protons and heavier nuclei), to study high energy gamma rays from astrophysical sources and to search for indirect dark-matter signatures. The main technical features of the detectors and a short review of the first preliminary results have been presented.

\section{References}

[1] J. Chang, Chinese J. Space Science 34 (2014) 550

[2] G. Ambrosi et al. (DAMPE Collaboration), "The DAMPE mission", to be submitted

[3] Z. Zhang et al., Nuclear Instrum. \& Meth. in Phys. Res. A836 (2016) 98

[4] V. Gallo et al., Proc. of the 34th Intern. Cosmic Ray Conference (2015) 1199

[5] P. Azzarello et al., Nuclear Instrum. \& Methods A 831 (2016) 378

[6] Z. Zhang et al., Nuclear Instrum. \& Methods A 836 (2016) 98

[7] T. Kobayashi et al., The Astrophys. Jour. 601 (2004) 340 ; D. Grasso et al., Astroparticle Physics 32 (2009) 140

[8] A.D. Panov et al., Bull. Russ. Acad. Sci. 71 (2007) 494

[9] H.S. Ahn et al., Astrophys. J. Lett. 714 (2010) L89; Y.S. Yoon et al., Astrophys. J. 728 (2011) 122

[10] O. Adriani et al., Science 332 (2011) 69

[11] L. Accardo et al., Phys. Rev. Lett. 114 (2015) 171103 